\title{
INTERPOLATION IN A CLASSICAL HILBERT SPACE OF ENTIRE FUNCTIONS(1)
}

\author{
BY \\ ROBERT M. YOUNG
}

\begin{abstract}
Let $H$ denote the Paley-Wiener space of entire functions of exponential type $\pi$ which belong to $L^{2}(-\infty, \infty)$ on the real axis. A sequence $\left\{\lambda_{n}\right\}$ of distinct complex numbers will be called an interpolating sequence for $H$ if $T H \supset l^{2}$, where $T$ is the mapping defined by $T f=\left\{f\left(\lambda_{n}\right)\right\}$. If in addition $\left\{\lambda_{n}\right\}$ is a set of uniqueness for $H$, then $\left\{\lambda_{n}\right\}$ is called a complete interpolating sequence. The following results are established. If $\operatorname{Re}\left(\lambda_{n+1}\right)$ $-\operatorname{Re}\left(\lambda_{n}\right) \geq \gamma>1$ and if the imaginary part of $\lambda_{n}$ is sufficiently small, then $\left\{\lambda_{n}\right\}$ is an interpolating sequence. If $\left|\operatorname{Re}\left(\lambda_{n}\right)-n\right| \leq L \leq(\log 2) / \pi(-\infty<n<\infty)$ and if the imaginary part of $\lambda_{n}$ is uniformly bounded, then $\left\{\lambda_{n}\right\}$ is a complete interpolating sequence and $\left\{e^{i \lambda_{0}}\right\}$ is a basis for $L^{2}(-\pi, \pi)$. These results are used to investigate interpolating sequences in several related spaces of entire functions of exponential type.
\end{abstract}

Introduction. Let $H$ denote the Paley-Wiener space of entire functions $f$ of exponential type $\pi$ for which

$$
\|f\|=\left[\int_{-\infty}^{\infty}|f(x)|^{2} d x\right]^{1 / 2}<\infty .
$$

A sequence $\left\{\lambda_{n}\right\}$ of distinct complex numbers will be called an interpolating sequence for $H$ if corresponding to each sequence $\left\{c_{n}\right\}$ in $l^{2}$ there is at least one function $f$ in $H$ satisfying $f\left(\lambda_{n}\right)=c_{n}(-\infty<n<\infty)$. (Unless otherwise stated, the term sequence in this paper will always mean a two-sided sequence.)

In $\$ \S 2$ and 3 of this paper we study both necessary and sufficient conditions for a sequence $\left\{\lambda_{n}\right\}$ to be interpolating. For the most part we require that the $\lambda_{n}$ lie in a strip parallel to the real axis. Under this condition we show that $l^{2}$ is the natural sequence space to interpolate with functions in $H$ and a necessary condition for interpolation is that the $\lambda_{n}$ be separated, that is, $\left|\lambda_{n}-\lambda_{k}\right| \geq \gamma$ for some constant $\gamma>0$ and all $n \neq k$.

A classical theorem of Paley and Wiener [8, p. 13] shows that the Fourier transform of every function in $H$ vanishes almost everywhere outside $(-\pi, \pi)$. Thus $f$ belongs to $H$ if and only if it is of the form

$$
f(z)=\frac{1}{2 \pi} \int_{-\pi}^{\pi} g(t) e^{i z t} d t
$$

Presented to the Society, January 17, 1972; received by the editors January 30, 1973.

AMS (MOS) subject classifications (1970). Primary 30A80; Secondary 42A80.

Key words and phrases. Paley-Wiener space, interpolating sequence, complete interpolating sequence, nonharmonic Fourier series expansion, entire functions of exponential type.

(1) This article is part of the author's doctoral dissertation at the University of Michigan under the supervision of Professor Douglas G. Dickson. The author wishes to thank Professor Dickson for his constant help and encouragement. 
for some $g$ in $L^{2}(-\pi, \pi)$. It follows that $\left\{\lambda_{n}\right\}$ is an interpolating sequence if and only if the trigonometric moment problem

$$
c_{n}=\frac{1}{2 \pi} \int_{-\pi}^{\pi} g(t) e^{i \lambda_{n} t} d t \quad(-\infty<n<\infty)
$$

has a solution $g$ in $L^{2}(-\pi, \pi)$ whenever $\Sigma\left|c_{n}\right|^{2}<\infty$. The following result was first established by Boas [3] and later reproved in a more abstract setting by $N$. Bari [1]. (See also [10].)

Lemma 1. A necessary and sufficient condition that the trigonometric moment problem (2) have a solution in $L^{2}(-\pi, \pi)$ for every square summable sequence $\left\{c_{n}\right\}$ is that the inequality

$$
A \sum\left|a_{n}\right|^{2} \leq \frac{1}{2 \pi} \int_{-\pi}^{\pi}\left|\Sigma a_{n} e^{i \lambda_{n} t}\right|^{2} d t
$$

holds for some constant $A>0$ and all finite sequences $\left\{a_{n}\right\}$.

Several years earlier, Ingham [7] had established the validity of (3) in the special case in which the $\lambda_{n}$ are real and satisfy the separation condition $\lambda_{n+1}-\lambda_{n} \geq \gamma>1(-\infty<n<\infty)$. He showed that his result is the best possible, in the sense that $\gamma$ cannot be taken equal to 1 , by demonstrating that the sequence $\left\{\lambda_{n}\right\}$ given by

$$
\lambda_{n}=n-\frac{1}{4}, \quad \lambda_{n}=-\lambda_{n} \quad(n=1,2, \ldots)
$$

does not satisfy (3) for any positive $A$. By modifying Ingham's proof, we are able to show that $\left\{\lambda_{n}\right\}$ is an interpolating sequence whenever

$$
\operatorname{Re}\left(\lambda_{n+1}\right)-\operatorname{Re}\left(\lambda_{n}\right) \geq \gamma>1 \quad(-\infty<n<\infty)
$$

and the imaginary part of $\lambda_{n}$ is sufficiently small. As a corollary we show that if $\left\{\lambda_{n}\right\}$ is a sequence of points lying in a strip parallel to the real axis and if $\operatorname{Re}\left(\lambda_{n+1}\right)-\operatorname{Re}\left(\lambda_{n}\right) \rightarrow \infty$ as $n \rightarrow \pm \infty$, then for each $\left\{c_{n}\right\}$ in $l^{2}$ and each $\tau>0$ there exists an entire function $f$ of exponential type $\tau$, square integrable on the real axis, for which $f\left(\lambda_{n}\right)=c_{n}(-\infty<n<\infty)$.

In their classic treatise, Paley and Wiener showed [8, p.115] that every sequence of real numbers $\left\{\lambda_{n}\right\}$ which are close to the integers in the sense that $\left|\lambda_{n}-n\right| \leq L<1 / \pi^{2}(-\infty<n<\infty)$ is an interpolating sequence. Moreover, they showed that every function $g$ in $L^{2}(-\pi, \pi)$ has a nonharmonic Fourier series expansion

$$
g(t)=\lim _{N \rightarrow \infty} . \sum_{-N}^{N} c_{n} e^{i \lambda_{n} t}
$$

with $\Sigma\left|c_{n}\right|^{2}<\infty$. These results were improved by Duffin and Eachus [5] who showed that $\lambda_{n}$ can be complex and that the constant $1 / \pi^{2}$ can be replaced by $(\log 2) / \pi$. In the present paper we show that $\left\{\lambda_{n}\right\}$ is an interpolating sequence 
whenever $\left|\operatorname{Re}\left(\lambda_{n}\right)-n\right| \leq L<(\log 2) / \pi(-\infty<n<\infty)$ and the imaginary part of $\lambda_{n}$ is uniformly bounded, and that under these conditions every function in $L^{2}(-\pi, \pi)$ has the representation (4) with some sequence $\left\{c_{n}\right\}$ in $l^{2}$.

The main results of $\$ \S 2$ and 3 are used in $\$ 4$ to investigate interpolating sequences in several related spaces of entire functions of exponential type. Specifically, we consider the spaces $E_{\tau}^{p}$ of entire functions of exponential type $\tau$ which belong to $L^{p}(-\infty, \infty)$ on the real axis. We restrict attention mainly to the special cases $p=1$ and $p=\infty$.

1. Background material. Under the inner product

$$
(f, g)=\int_{-\infty}^{\infty} f(x) \overline{g(x)} d x,
$$

$H$ is a functional Hilbert space with the reproducing kernel

$$
K(\xi, z)=\sin \pi(\xi-\bar{z}) / \pi(\xi-\bar{z}) .
$$

The functions $K(\xi, n),-\infty<n<\infty$, form a complete orthonormal system, so that for each $f$ in $H$

$$
f(z)=\sum_{-\infty}^{\infty} f(n) \frac{\sin \pi(z-n)}{\pi(z-n)} .
$$

By Parseval's formula

$$
\|f\|^{2}=\sum_{-\infty}^{\infty}|f(n)|^{2} \quad(f \in H) .
$$

If $f$ is given by (1), then $g$ is the Fourier transform of $f$ and Plancherel's theorem . gives

$$
\int_{-\infty}^{\infty}|f(x)|^{2} d x=\frac{1}{2 \pi} \int_{-\pi}^{\pi}|g(x)|^{2} d x .
$$

It follows easily from (1) and (6) that

$$
|f(x+i y)| \leq e^{\pi|y|}\|f\|
$$

for every $f$ belonging to $H$. Similar estimates show that $H$ is closed under differentiation and that

$$
\left\|f^{\prime}\right\| \leq \pi\|f\| \quad(f \in H) .
$$

2. Interpolation in $H$. Let $\left\{\lambda_{n}\right\}$ be a sequence of distinct complex numbers. For each $f$ in $H$ we define $T f$ to be the sequence $T f=\left\{f\left(\lambda_{n}\right)\right\}(-\infty<n<\infty)$.

Definition. The sequence $\left\{\lambda_{n}\right\}$ is said to be an interpolating sequence for $H$ if $T H \supset l^{2}$. It is not difficult to show that if $T H \supset l^{2}$, then the unit ball in $l^{2}$ can be interpolated in a uniformly bounded way. In fact, we have the following stronger result. For a proof see $[9$, p. 19]. 
Lemma 2. Let $X$ be a Banach space, $\left\{\mu_{n}\right\}$ a sequence in the dual space $X^{\prime}$ and $T$ the mapping on $X$ defined by $T x=\left\{\mu_{n}(x)\right\}$. If $T X \supset l^{p}$ for some $p, 1 \leq p \leq \infty$, then TM covers the unit ball of $l^{p}$ for some bounded subset $M$ of $X$.

Definition. The sequence $\left\{\lambda_{n}\right\}$ is said to be separated if there is a constant $\gamma$ such that $\left|\lambda_{n}-\lambda_{k}\right| \geq \gamma>0(n \neq k)$.

It is well known $\left[4\right.$, p. 101] that if the $\lambda_{n}$ are real and separated and if $f$ is an entire function of exponential type $\tau$ belonging to $L^{p}(-\infty, \infty)$ on the real axis $(0<p<\infty)$, then

$$
\sum\left|f\left(\lambda_{n}\right)\right|^{p} \leq B \int_{-\infty}^{\infty}|f(x)|^{p} d x,
$$

where $B$ depends only on $p, \tau$ and the separation constant $\gamma$. It is no more difficult to establish the following result, which we state without proof.

Lemma 3. Let $\left\{\lambda_{n}\right\}$ be a complex sequence satisfying

$$
\left|\operatorname{Im}\left(\lambda_{n}\right)\right| \leq \alpha, \quad\left|\lambda_{n}-\lambda_{k}\right| \geq \gamma \quad(n \neq k)
$$

for some positive constants $\alpha$ and $\gamma$. If $f$ is an entire function of exponential type $\tau$, belonging to $L^{p}(-\infty, \infty)$ on the real axis, then

$$
\sum\left|f\left(\lambda_{n}\right)\right|^{p} \leq B \int_{-\infty}^{\infty}|f(x)|^{p} d x,
$$

where $B=B(p, \tau, \gamma, \alpha)$.

Proposition 1. Let $\left\{\lambda_{n}\right\}$ be a sequence of points lying in a strip parallel to the real axis. If $T H \supset l^{1}$, then $\left\{\lambda_{n}\right\}$ is separated.

Proof. Since point-evaluations are continuous linear functionals on $H$, Lemma 2 shows that there are functions $f_{n}$ in $H$ and a constant $M>0$ such that $f_{n}\left(\lambda_{k}\right)=\delta_{n k},\left\|f_{n}\right\| \leq M$ (for all $\left.n, k\right)$. For $n \neq k$, we have

$$
1=f_{n}\left(\lambda_{n}\right)-f_{n}\left(\lambda_{k}\right)=\int_{\lambda_{k}}^{\lambda_{n}} f_{n}^{\prime}(z) d z
$$

so that

$$
1 \leq \int_{\lambda_{k}}^{\lambda_{n}}\left|f_{n}^{\prime}(z)\right||d z| \leq \sup \left|f_{n}^{\prime}(z)\right| \cdot\left|\lambda_{n}-\lambda_{k}\right|
$$

where the supremum is taken over the straight line segment from $\lambda_{k}$ to $\lambda_{n}$. If $\left|\operatorname{Im}\left(\lambda_{n}\right)\right| \leq \alpha$, then (7), (8) show that

$$
\begin{aligned}
1 & \leq e^{\pi \alpha}\left\|f_{n}^{\prime}\right\| \cdot\left|\lambda_{n}-\lambda_{k}\right| \\
& \leq \pi e^{\pi \alpha}\left\|f_{n}\right\| \cdot\left|\lambda_{n}-\lambda_{k}\right| \\
& \leq M \pi e^{\pi \alpha}\left|\lambda_{n}-\lambda_{k}\right|,
\end{aligned}
$$

and the result follows with $\gamma=e^{-\pi \alpha} / M \pi$. 
Corollary 1. Let $\left\{\lambda_{n}\right\}$ be a sequence of points lying in a strip parallel to the real axis. If $T H \supset l^{1}$, then $T H \subset l^{2}$.

Since separation is a necessary condition for a sequence of real numbers to be interpolating, the problem arises to determine if there is a constant $\gamma$ with the property that $\left\{\lambda_{n}\right\}$ is an interpolating sequence whenever $\lambda_{n+1}-\lambda_{n} \geq \gamma$. The following result will show that $\left\{\lambda_{n}\right\}$ is interpolating whenever $\gamma>1$. The proof is a simple extension of an argument given by Ingham [7].

Lemma 4. Let $f(t)=\sum_{-N}^{N} a_{n} e^{i \lambda_{n} t}$ where the $\lambda_{n}$ satisfy

$$
\operatorname{Re}\left(\lambda_{n+1}\right)-\operatorname{Re}\left(\lambda_{n}\right) \geq \gamma>1, \quad\left|\operatorname{Im}\left(\lambda_{n}\right)\right| \leq \alpha .
$$

Then

$$
A \sum\left|a_{n}\right|^{2} \leq \int_{-\pi}^{\pi}|f(t)|^{2} d t
$$

where $A=4\left[1 /\left(1+16 \alpha^{2}\right)-e^{2 \pi \alpha} / \gamma^{2}\right]$.

Proof. If $k$ belongs to $L^{1}(-\infty, \infty)$ and $K(z)=\int_{-\infty}^{\infty} k(t) e^{i z t} d t$, then

$$
\int_{-\infty}^{\infty} k(t)|f(t)|^{2} d t=\sum_{m, n} a_{m} \bar{a}_{n} K\left(\lambda_{m}-\bar{\lambda}_{n}\right)
$$

Letting

$$
\begin{aligned}
k(t) & =\cos \frac{1}{2} t, & & |t| \leq \pi, \\
& =0, & & |t|>\pi,
\end{aligned}
$$

we get $K(z)=4(\cos \pi z) /\left(1-4 z^{2}\right)$. Since $|k(t)| \leq 1$, it follows from (10) that

$$
\int_{-\pi}^{\pi}|f(t)|^{2} d t \geq\left.\left|\sum_{n}\right| a_{n}\right|^{2} K\left(\lambda_{n}-\bar{\lambda}_{n}\right)|-| \sum_{m, n}^{\prime} a_{m} \bar{a}_{n} K\left(\lambda_{m}-\bar{\lambda}_{n}\right) \mid,
$$

where the prime in the summation denotes omission of the term $m=n$. The remainder of the proof is devoted to obtaining suitable estimates for the two sums in absolute value above. Since, by (9), $\left|\lambda_{m}-\bar{\lambda}_{n}\right| \geq|m-n| \gamma>1(m \neq n)$ and since $|\cos (x+i y)| \leq e^{|y|}$, we have

$$
\begin{aligned}
\sum_{m}^{\prime}\left|K\left(\lambda_{m}-\bar{\lambda}_{n}\right)\right| & \leq 4 e^{2 \pi \alpha} \sum_{m}^{\prime} \frac{1}{4(m-n)^{2} \gamma^{2}-1} \\
& <\frac{8 e^{2 \pi \alpha}}{\gamma^{2}} \sum_{r=1}^{\infty} \frac{1}{4 n^{2}-1}=\frac{4 e^{2 \pi \alpha}}{\gamma^{2}} .
\end{aligned}
$$

Since $2\left|a_{m} a_{n}\right| \leq\left|a_{m}\right|^{2}+\left|a_{n}\right|^{2}$, we get

$$
\sum_{m, n}^{\prime} a_{m} \bar{a}_{n} K\left(\lambda_{m}-\bar{\lambda}_{n}\right)=\theta \sum_{m, n}^{\prime} \frac{\left|a_{m}\right|^{2}+\left|a_{n}\right|^{2}}{2}\left|K\left(\lambda_{m}-\bar{\lambda}_{n}\right)\right|
$$

where $|\theta| \leq 1$, and since $|K(z)|=|K(-\bar{z})|$, 


$$
\begin{aligned}
\sum_{m, n}^{\prime} a_{m} \bar{a}_{n} K\left(\lambda_{m}-\bar{\lambda}_{n}\right) & =\theta \sum_{n}\left|a_{n}\right|^{2}\left\{\sum_{m}^{\prime}\left|K\left(\lambda_{m}-\bar{\lambda}_{n}\right)\right|\right\} \\
& <\frac{4 e^{2 n a}}{\gamma^{2}} \sum_{n}\left|a_{n}\right|^{2} .
\end{aligned}
$$

If we set $\beta_{n}=\operatorname{Im}\left(\lambda_{n}\right)$, then

$$
K\left(2 i \beta_{n}\right)=\frac{4 \cosh 2 \pi \beta_{n}}{1+16 \beta_{n}^{2}} \geq \frac{4}{1+16 \alpha^{2}},
$$

and hence

$$
\sum_{n}\left|a_{n}\right|^{2} K\left(\lambda_{n}-\bar{\lambda}_{n}\right) \geq \frac{4}{1+16 \alpha^{2}} \sum\left|a_{n}\right|^{2}
$$

and the proof is complete.

Theorem 1. Let $\left\{\lambda_{n}\right\}$ be a complex sequence satsifying

$$
\begin{aligned}
\operatorname{Re}\left(\lambda_{n+1}\right)-\operatorname{Re}\left(\lambda_{n}\right) & \geq \gamma>1, \\
\left|\operatorname{Im}\left(\lambda_{n}\right)\right| & \leq \alpha \quad(-\infty<n<\infty) .
\end{aligned}
$$

Then $\left\{\lambda_{n}\right\}$ is an interpolating sequence for all sufficiently small values of $\alpha$.

Proof. The result follows immediately from Lemmas 1 and 4 since the value of $A$ given in the statement of Lemma 4 approaches $4\left(1-1 / \gamma^{2}\right)>0$ as $\alpha \rightarrow 0$.

Remark. Shapiro and Shields have shown $\left[10\right.$, p. 532] that if $\left\{\lambda_{n}\right\}$ is a separated sequence of real numbers and if $\Sigma\left|c_{n}\right|^{2}<\infty$, then for each $\alpha>0$ there corresponds a function $f$ analytic in the strip $D:|y|<\alpha$, with finite area norm

$$
\iint_{D}|f(z)|^{2} d x d y<\infty
$$

such that $f\left(\lambda_{n}\right)=c_{n}(-\infty<n<\infty)$. Since (11) is satisfied for each function in $H$, an obvious extension of Theorem 1 shows that $f$ may in fact be chosen to be entire of exponential type.

Theorem 2. Let $\left\{\lambda_{n}\right\}$ be a sequence of distinct points lying in a strip parallel to the real axis and suppose that $\operatorname{Re}\left(\lambda_{n+1}\right)-\operatorname{Re}\left(\lambda_{n}\right) \rightarrow \infty, n \rightarrow \pm \infty$. For each $\tau>0$ and each sequence $\left\{c_{n}\right\}$ in $l^{2}$ there exists an entire function $f$ of exponential type $\tau$, belonging to $L^{2}(-\infty, \infty)$ on the real axis, for which $f\left(\lambda_{n}\right)=c_{n}(-\infty<n<\infty)$.

Proof. Fix $\tau>0$ and let $H_{\tau}$ denote the space of entire functions of exponential type $\tau$ which belong to $L^{2}(-\infty, \infty)$ on the real axis. It follows from Theorem 1 that there exists a smallest integer $N \geq 0$ with the property that for each sequence $\left\{c_{n}\right\}$ in $l^{2}$ there corresponds at least one function $f$ in $H_{\tau}$ such that $f\left(\lambda_{n}\right)=c_{n},|n| \geq N$. If $N=0$ there is nothing to show, so suppose that $N \geq 1$. Choose $g$ in $H_{\tau}$ such that 


$$
\begin{aligned}
& g\left(\lambda_{N}\right)=1, \quad|n| \geq N, n \neq N . \\
& g\left(\lambda_{n}\right)=0, \quad
\end{aligned}
$$

We begin by constructing a function $h$ in $H_{r}$ such that

$$
h\left(\lambda_{N-1}\right)=1, \quad h\left(\lambda_{n}\right)=0, \quad|n| \geq N .
$$

If $g\left(\lambda_{N-1}\right)=0$, then for suitable constants $c$ and $m \geq 1$ the function

$$
h(z)=\left[\frac{g(z)}{c\left(z-\lambda_{N-1}\right)^{m}}\right]\left[\frac{z-\lambda_{N}}{\lambda_{N-1}-\lambda_{N}}\right]
$$

satisfies (12). If $g\left(\lambda_{N-1}\right) \neq 0$, then $h$ must be obtained by a different method. By a theorem of Titchmarsh [11] the zeros of $g$ have a positive density, where it is understood that multiple zeros are counted as many times as their multiplicity warrants. It is easily shown that $\left\{\lambda_{n}\right\}$ has density zero, so that some zero $\lambda$ of $g$ is either not in the sequence or else is in the sequence and is a multiple zero of $g$. In either case, the function

$$
h(z)=\frac{\lambda_{N-1}-\lambda}{\lambda_{N-1}-\lambda_{N}}\left[\frac{g(z)}{g\left(\lambda_{N-1}\right)} \cdot \frac{z-\lambda_{N}}{z-\lambda}\right]
$$

satisfies (12).

Now fix $\left\{c_{n}\right\}$ in $l^{2}$ and choose $f$ in $H_{\tau}$ such that $f\left(\lambda_{n}\right)=c_{n}(|n| \geq N)$. Let $g$ and $h$ be chosen as above and define

$$
f_{1}(z)=f(z)+\left[c_{N-1}-f\left(\lambda_{N-1}\right)\right] h(z) .
$$

Then $f_{1}$ belongs to $H_{\tau}$ and $f_{1}\left(\lambda_{n}\right)=c_{n}(|n| \geq N$ and $n=N-1)$. The same construction gives $f_{2} \in H_{\tau}$ such that $f_{2}\left(\lambda_{n}\right)=c_{n}(|n| \geq N-1)$. But this contradicts the choice of $N$; hence $N$ must be equal to zero, and the proof is complete.

Theorem 3. Let $\left\{\lambda_{n}\right\}, n=1,2, \ldots$, be an interpolating sequence. There exist positive numbers $\delta_{n}$ such that $\left\{\mu_{n}\right\}$ is an interpolating sequence whenever $\left|\lambda_{n}-\mu_{n}\right|$ $<\delta_{n}$.

Proof. It follows from Lemma 2 that there is a constant $M>0$ depending only on $\left\{\lambda_{n}\right\}$ with the property that for each sequence $\left\{c_{n}\right\}$ in the unit ball of $l^{2}$ there corresponds at least one function $f$ in $H$ for which $f\left(\lambda_{n}\right)=c_{n}(n=1,2, \ldots)$ and $\|f\| \leq M$.

Let $\mathscr{F}_{1}$ denote the family of all functions $f$ in $H$ for which $\|f\| \leq M$. Then (7) shows that the functions in $\mathscr{F}_{1}$ are uniformly bounded on compacta. It follows that $\mathscr{F}_{1}$ is equicontinuous on compacta and hence in particular on the disk $\left|z-\lambda_{1}\right| \leq 1$. Thus, if $0<\varepsilon_{1}<1$, there is a corresponding $\delta_{1}=\delta_{1}\left(\varepsilon_{1}\right)$ such that $\left|f(z)-f\left(\lambda_{1}\right)\right|<\varepsilon\left(\left|z-\lambda_{1}\right|<\delta_{1}\right)$, uniformly for all $f$ in $\mathscr{F}_{1}$. In addition, $\delta_{1}$ may be chosen small enough so that the disk $\left|z-\lambda_{1}\right|<\delta_{1}$ intersects $\left\{\lambda_{n}\right\}$ only at $\lambda_{1}$. 
We are going to show that if $\left|\lambda_{1}-\mu_{1}\right|<\delta_{1}$, then $\left\{\mu_{1}, \lambda_{2}, \lambda_{3}, \ldots\right\}$ is an interpolating sequence. Clearly, it will be enough to show that the unit ball of $l^{2}$ can be interpolated. More precisely, we will show that for each $\left\{c_{n}\right\}$ with $\Sigma\left|c_{n}\right|^{2} \leq 1$ there corresponds a function $F_{1}$ in $H$ for which

$$
\begin{aligned}
F_{1}\left(\mu_{1}\right) & =c_{1}, \\
F_{1}\left(\lambda_{n}\right) & =c_{n}, \\
\left\|F_{1}\right\| & \leq M /\left(1-\varepsilon_{1}\right),
\end{aligned}
$$

Fix $\mu_{1}$ with $\left|\lambda_{1}-\mu_{1}\right|<\delta_{1}$ and let $\Sigma\left|c_{n}\right|^{2} \leq 1$. There exists a function $g$ in $H$ such that

$$
g\left(\lambda_{n}\right)=c_{n}, \quad\|g\| \leq M, \quad n=1,2, \ldots .
$$

Since $g$ is in $\mathscr{F}_{1},\left|g\left(\mu_{1}\right)-g\left(\lambda_{1}\right)\right|<\varepsilon_{1}$. Also, there exists a function $f$ in $H$ such that

$$
\begin{aligned}
f\left(\lambda_{1}\right) & =1, \\
f\left(\lambda_{n}\right) & =0, \quad n=2,3, \ldots . \\
\|f\| & \leq M,
\end{aligned}
$$

Then $f$ is also in $\mathscr{F}_{1}$ so that $\left|f\left(\mu_{1}\right)-f\left(\lambda_{1}\right)\right|<\varepsilon_{1}$, and hence $\left|f\left(\mu_{1}\right)\right|>1-\varepsilon_{1}>0$. Now, set

$$
F_{1}(z)=g(z)+\left[c_{1}-g\left(\mu_{1}\right)\right] f(z) / f\left(\mu_{1}\right) .
$$

Clearly, $F_{1}$ is in $H, F_{1}\left(\mu_{1}\right)=c_{1}, F_{1}\left(\lambda_{n}\right)=c_{n}(n>1)$, and

$$
\begin{aligned}
\left\|F_{1}\right\| & \leq\|g\|+\frac{\mid c_{1}-g\left(\mu_{1}\right)}{\left|f\left(\mu_{1}\right)\right|}\|f\| \\
& \leq\|g\|+\frac{\left|g\left(\lambda_{1}\right)-g\left(\mu_{1}\right)\right|}{\left|f\left(\mu_{1}\right)\right|}\|f\| \\
& \leq M\left(1+\varepsilon_{1} /\left(1-\varepsilon_{1}\right)\right)=M /\left(1-\varepsilon_{1}\right) .
\end{aligned}
$$

The above argument can be repeated with $\left\{\lambda_{n}\right\}$ replaced by $\left\{\mu_{1}, \lambda_{2}, \lambda_{3}, \ldots\right\}$. Thus, we let $\mathscr{F}_{2}$ denote the family of all functions $f$ in $H$ for which $\|f\|$ $\leq M /\left(1-\varepsilon_{1}\right)$. Then $\mathscr{F}_{2}$ is equicontinuous on each compact set and for $0<\varepsilon_{2}$ $<1$ we find $\delta_{2}=\delta_{2}\left(\varepsilon_{1}, \varepsilon_{2}\right)$ such that

$$
\left|f(z)-f\left(\lambda_{2}\right)\right|<\varepsilon_{2} \quad\left(\left|z-\lambda_{2}\right|<\delta_{2}\right),
$$

uniformly for all $f$ in $\mathcal{F}_{2}$. We note that $\delta_{2}$ is independent of $\mu_{1}$ and may be chosen so that the disks $\left|z-\lambda_{1}\right|<\delta_{1}$ and $\left|z-\lambda_{2}\right|<\delta_{2}$ are disjoint and intersect $\left\{\lambda_{n}\right\}$ only at $\lambda_{1}$ and $\lambda_{2}$, respectively. Just as before, we show that whenever $\left|\mu_{2}-\lambda_{2}\right|$ $<\delta_{2}$, the sequence $\left\{\mu_{1}, \mu_{2}, \lambda_{3}, \lambda_{4}, \ldots\right\}$ is interpolating, and that for each sequence $\left\{c_{n}\right\}$ in the unit ball of $l^{2}$ there corresponds a function $F_{2}$ in $H$ for which 


$$
\begin{aligned}
& F_{2}\left(\mu_{n}\right)=c_{n}, \quad n=1,2, \\
& F_{2}\left(\lambda_{n}\right)=c_{n}, \quad n>2, \\
&\left\|F_{2}\right\| \leq M /\left(1-\varepsilon_{1}\right)\left(1-\varepsilon_{2}\right) .
\end{aligned}
$$

The above process may be iterated. Thus, given a sequence $\left\{\varepsilon_{n}\right\}, 0<\varepsilon_{n}<1$, we obtain a corresponding sequence $\left\{\delta_{n}\right\}, \delta_{n}=\delta_{n}\left(\varepsilon_{1}, \varepsilon_{2}, \ldots, \varepsilon_{n}\right)>0$, with the property that for each positive integer $N$ the sequence $\left\{\mu_{1}, \mu_{2}, \ldots, \mu_{N}, \lambda_{N+1}\right.$, $\left.\lambda_{N+2}, \ldots\right\}$ is interpolating whenever $\left|\lambda_{n}-\mu_{n}\right|<\delta_{n}(n=1,2, \ldots, N)$, and such that for every sequence $\left\{c_{n}\right\}$ with $\sum\left|c_{n}\right|^{2} \leq 1$ there exists a function $F_{N}$ in $H$ for which

$$
\begin{aligned}
F_{N}\left(\mu_{n}\right) & =c_{n}, \quad n=1,2, \ldots, N, \\
F_{N}\left(\lambda_{n}\right) & =c_{n}, \quad n>N, \\
\left\|F_{N}\right\| & \leq M \frac{1}{1-\varepsilon_{1}} \frac{1}{1-\varepsilon_{2}} \cdots \frac{1}{1-\varepsilon_{N}} .
\end{aligned}
$$

Now, choose $\left\{\varepsilon_{n}\right\}, 0<\varepsilon_{n}<1$, so that

$$
\varepsilon=\sum_{n=1}^{\infty} \frac{\varepsilon_{n}}{1-\varepsilon_{n}}<\infty,
$$

and let the corresponding sequence $\left\{\delta_{n}\right\}$ be determined as above. Fix $\left\{\mu_{n}\right\}$ with $\left|\lambda_{n}-\mu_{n}\right|<\delta_{n}(n=1,2, \ldots)$ and let $\left\{c_{n}\right\}$ belong to the unit ball of $l^{2}$. For each positive integer $N$ there exists a function $F_{N}$ in $H$ such that

$$
\begin{aligned}
F_{N}\left(\mu_{n}\right) & =c_{n}, \quad n=1,2, \ldots, N \\
\left\|F_{N}\right\| & \leq M \prod_{n=1}^{N}\left(1+\frac{\varepsilon_{n}}{1-\varepsilon_{n}}\right) \\
& \leq M \exp \left[\sum_{n=1}^{\infty} \frac{\varepsilon_{n}}{1-\varepsilon_{n}}\right]=M e^{\varepsilon}
\end{aligned}
$$

Since $\left\|F_{N}\right\|$ is uniformly bounded, a subsequence of $\left\{F_{N}\right\}$ will converge weakly to a function $F$ in $H$ for which $F\left(\mu_{n}\right)=c_{n}(n=1,2, \ldots)$. Thus, the unit ball of $l^{2}$ can be interpolated and the proof is complete.

3. Uniqueness: complete interpolatng sequences.

Proposition 2. Let $\left\{\lambda_{n}\right\}, n=1,2, \ldots$, be an interpolating sequence. Each of the following statements implies the others.

(i) The set of relations $f \in H$ and $f\left(\lambda_{n}\right)=0(n=1,2, \ldots)$ imply that $f \equiv 0$, that is, $\left\{\lambda_{n}\right\}$ is a set of uniqueness for $H$.

(ii) The exponentials $\left\{e^{i \lambda_{n} t}\right\}$ are complete in $L^{2}(-\pi, \pi)$.

(iii) The sequence $\left\{\lambda_{n}\right\}$ is contained in no larger interpolating sequence. 
Proof. It follows immediately from the Paley-Wiener representation (1) that (i) and (ii) are equivalent. Since (ii) clearly implies (iii) it remains only to show that (iii) implies (ii).

Suppose then that $\left\{e^{i \lambda_{n} t}\right\}$ is not complete in $L^{2}(-\pi, \pi)$ and let $\lambda_{0}$ be distinct from the $\lambda_{n}$. We show that $\left\{\lambda_{n}\right\}, n=0,1,2, \ldots$, is an interpolating sequence. Since $\left\{e^{i \lambda_{n} t}\right\}$ is incomplete there is a function $g$ in $L^{2}(-\pi, \pi), g \neq 0$, such that

$$
\int_{-\pi}^{\pi} g(t) e^{i \lambda_{n} t} d t=0, \quad n=1,2, \ldots
$$

Setting $h(z)=\int_{-\rightarrow}^{\pi} g(t) e^{i z t} d t$, we have $h \in H, h \not \equiv 0$, and $h\left(\lambda_{n}\right)=0(n=1$, $2, \ldots)$. If $h\left(\lambda_{0}\right) \neq 0$, we set $F(z)=h(z) / h\left(\lambda_{0}\right)$, while if $h\left(\lambda_{0}\right)=0$, we take $F(z)=h(z) / A\left(z-\lambda_{0}\right)^{m}$, where $A$ and $m$ are chosen so that $F\left(\lambda_{0}\right)=1$. In either case, $F\left(\lambda_{0}\right)=1$ and $F\left(\lambda_{n}\right)=0(n=1,2, \ldots)$.

Fix $\left\{c_{n}\right\}, n=0,1,2, \ldots$, in $l^{2}$. There is a function $G$ in $H$ with $G\left(\lambda_{n}\right)=c_{n}$ $(n=1,2, \ldots)$. Let

$$
f(z)=G(z)+\left[c_{0}-G\left(\lambda_{0}\right)\right] F(z) .
$$

Then $f$ is in $H$ and $f\left(\lambda_{n}\right)=c_{n}(n=0,1,2, \ldots)$.

Definition. An interpolating sequence satisfying any one of the conditions listed in Proposition 2 will be called a complete interpolating sequence.

Theorem 4. Let $\left\{\lambda_{n}\right\}$ be a sequence of distinct points lying in a strip parallel to the real axis. If $\left\{\operatorname{Re}\left(\lambda_{n}\right)\right\}$ is a complete interpolating sequence, then $\left\{\lambda_{n}\right\}$ is a complete interpolating sequence.

The proof of Theorem 4 requires the following lemma.

Lemma 5. Let $\lambda_{n}=\alpha_{n}+i \beta_{n}$, where $\alpha_{n}$ and $\beta_{n}$ are real and satisfy

$$
\alpha_{n+1}-\alpha_{n} \geq \gamma>0, \quad\left|\beta_{n}\right| \leq \beta, \quad-\infty<n<\infty .
$$

If $\left\{e^{i \lambda_{n} t}\right\}$ is complete in $L^{2}(-\pi, \pi)$, then $\left\{e^{i a_{n} t}\right\}$ is also complete in $L^{2}(-\pi, \pi)$.

Proof of Lemma 5. An equivalent problem is to show that the completeness of $\left\{e^{i\left(\lambda_{n}+i\right) t}\right\}$ implies that of $\left\{e^{i\left(a_{n}+i\right) t}\right\}$. For this it is enough to show that the only function in $H$ which vanishes at every point $\alpha_{n}+i$ is identically zero. Arguing by contradiction, we suppose that for some $f$ in $H, f \neq 0, f\left(\alpha_{n}+i\right)=0(-\infty<n$ $<\infty)$. Without any loss of generality we may suppose that no $\alpha_{n}$ is an integer and that $f(0)=1$. We are going to exhibit a function $g$ in $H$ with $g\left(\lambda_{n}+i\right)=0$ $(-\infty<n<\infty)$ and $g(0)=1$, thereby contradicting the completeness of $\left\{e^{i\left(\lambda_{n}+i\right) t}\right\}$. Set

$$
f_{N}(z)=f(z) \prod_{n=-N}^{N} \frac{1-z /\left(\lambda_{n}+i\right)}{1-z /\left(\alpha_{n}+i\right)}, \quad N=1,2, \ldots
$$

For each $N$ we have $f_{N} \in H, f_{N}\left(\lambda_{n}+i\right)=0(|n| \leq N)$, and $f_{N}(0)=1$. By (5), 


$$
\left\|f_{N}\right\|^{2}=\sum_{k=-\infty}^{\infty}\left|f_{N}(k)\right|^{2}=\sum_{k=-\infty}^{\infty}|f(k)|^{2}\left[\prod_{n=-N}^{N}\left|\frac{1-k /\left(\lambda_{n}+i\right)}{1-k /\left(\alpha_{n}+i\right)}\right|^{2}\right] \text {. }
$$

We show that the products

$$
\stackrel{\prod}{n}=-N_{N}^{N}\left|\frac{1-k /\left(\lambda_{n}+i\right)}{1-k /\left(\alpha_{n}+i\right)}\right|^{2}=\prod_{n=-N}^{N}\left|\frac{\alpha_{n}+i}{\lambda_{n}+i}\right|^{2}\left|\frac{\lambda_{n}+i-k}{\alpha_{n}+i-k}\right|^{2}
$$

are uniformly bounded in $N$ and $k$. Simple calculations show that

$$
\begin{aligned}
\left|\frac{\alpha_{n}+i}{\lambda_{n}+i}\right|^{2} & =\frac{\alpha_{n}^{2}+1}{\alpha_{n}^{2}+\left(\beta_{n}+1\right)^{2}}=1-\frac{\beta_{n}^{2}+2 \beta_{n}}{\alpha_{n}^{2}+\left(\beta_{n}+1\right)^{2}} \leq 1+\frac{\beta_{n}^{2}+2\left|\beta_{n}\right|}{\alpha_{n}^{2}+\left(\beta_{n}+1\right)^{2}} \\
& \leq 1+\frac{\beta^{2}+2 \beta}{\alpha_{n}^{2}}=1+\frac{A}{\alpha_{n}^{2}},
\end{aligned}
$$

and

$$
\begin{aligned}
\left|\frac{\lambda_{n}+i-k}{\alpha_{n}+i-k}\right|^{2} & =\frac{\left(\alpha_{n}-k\right)^{2}+\left(\beta_{n}+1\right)^{2}}{\left(\alpha_{n}-k\right)^{2}+1} \leq \frac{\left(\alpha_{n}-k\right)^{2}+(\beta+1)^{2}}{\left(\alpha_{n}-k\right)^{2}+1} \\
& =1+\frac{\beta^{2}+2 \beta}{\left(\alpha_{n}-k\right)^{2}+1}=1+\frac{B}{\left(\alpha_{n}-k\right)^{2}+1} .
\end{aligned}
$$

Therefore

$$
\begin{aligned}
\prod_{n=-N}^{N}\left|\frac{1-k /\left(\lambda_{n}+i\right)}{1-k /\left(\alpha_{n}+i\right)}\right|^{2} & \leq \prod_{n=-\infty}^{\infty}\left[1+\frac{A}{\alpha_{n}^{2}}\right]\left[1+\frac{B}{\left(\alpha_{n}-k\right)^{2}+1}\right] \\
& \leq \exp \left\{\sum_{n=-\infty}^{\infty}\left[\frac{A}{\alpha_{n}^{2}}+\frac{B}{\left(\alpha_{n}-k\right)^{2}+1}\right]\right\} .
\end{aligned}
$$

Since $\left\{\alpha_{n}\right\}$ is separated and no $\alpha_{n}$ vanishes, the series $\Sigma \alpha_{n}^{-2}$ converges and

$$
\sum_{n=-\infty}^{\infty} \frac{1}{\left(\alpha_{n}-k\right)^{2}+1} \leq 2+\sum_{n=1}^{\infty} \frac{1}{(n \gamma)^{2}} \quad(-\infty<k<\infty) .
$$

It follows that $\sup \left\|f_{N}\right\|<\infty$, so that a subsequence of $\left\{f_{N}\right\}$ converges weakly to a function $g$ in $H$ for which $g\left(\lambda_{n}+i\right)=0(-\infty<n<\infty)$ and $g(0)=1$.

Proof of Theorem 4. Let $\lambda_{n}=\alpha_{n}+i \beta_{n}$. Since $\left\{\alpha_{n}\right\}$ is a complete interpolating sequence, it follows from Lemma 3 and Proposition 1 that the mapping $T: H \rightarrow l^{2}$ given by $T f=\left\{f\left(\alpha_{n}\right)\right\}$ is continuous, one-to-one, and onto. By the open mapping theorem, $T$ has a continuous inverse. Thus, there exists a positive constant $A$ such that

$$
A\|f\|^{2} \leq \Sigma\left|f\left(\alpha_{n}\right)\right|^{2} \quad(f \in H) .
$$

By a theorem of Duffin and Schaeffer $[6$, p. 355] we have

$$
B\|f\|^{2} \leq \Sigma\left|f\left(\lambda_{n}\right)\right|^{2}
$$


for some constant $B>0$ and all $f$ in $H$. Now there exist functions $g_{n}$ in $H$ such that $g_{n}\left(\alpha_{k}\right)=\delta_{n k}$. It follows from Lemma 5 that for each $k$ the sequence of functions $\left\{e^{i \lambda_{n} t}\right\}_{n \neq k}$ is incomplete in $L^{2}(-\pi, \pi)$. Therefore, we can find functions $f_{n}$ in $H$ such that $f_{n}\left(\lambda_{k}\right)=\delta_{n k}$. Fix $\left\{c_{n}\right\}$ in $l^{2}$ and set $F_{N}(z)=\sum_{-N}^{N} c_{n} f_{n}(z)$ $(N=1,2, \ldots)$. Since $F_{N}\left(\lambda_{k}\right)$ is equal to $c_{k}$ when $|k| \leq N$ and has the value 0 for $|k|>N,(13)$ gives

$$
\left\|F_{N}\right\|^{2} \leq \frac{1}{B} \sum_{k=-\infty}^{\infty}\left|F_{N}\left(\lambda_{k}\right)\right|^{2} \leq \frac{1}{B} \sum_{-\infty}^{\infty}\left|c_{k}\right|^{2}
$$

so that a subsequence of $\left\{F_{N}\right\}$ converges weakly to a function $F \in H$ for which $F\left(\lambda_{k}\right)=c_{k}(-\infty<k<\infty)$.

Corollary 2. Let $\left\{\lambda_{n}\right\}$ be a sequence of points lying in a strip parallel to the real axis, and suppose that

$$
\left|\operatorname{Re}\left(\lambda_{n}\right)-n\right| \leq L<(\log 2) / \pi \quad(-\infty<n<\infty)
$$

Then $\left\{\lambda_{n}\right\}$ is a complete interpolating sequence.

Proof. It was shown by Duffin and Eachus [5] that the inequality

$$
\left\|\sum c_{n}\left(e^{i \operatorname{Re}\left(\lambda_{n}\right) t}-e^{i n t}\right)\right\|_{D^{2}(-\pi, n)}^{2} \leq \theta^{2} \sum\left|c_{n}\right|^{2}
$$

holds for some constant $\theta, 0 \leq \theta<1$, and every sequence $\left\{c_{n}\right\}$ in $l^{2}$. By a theorem of Paley and Wiener [8, p. 100], $\left\{\operatorname{Re}\left(\lambda_{n}\right)\right\}$ is a complete interpolating sequence. The result then follows from Theorem 4.

Theorem 5. Let $\left\{\lambda_{n}\right\}$ be a sequence of points lying in a strip parallel to the real axis. If $\left\{\lambda_{n}\right\}$ is a complete interpolating sequence, then each function in $L^{2}(-\pi, \pi)$ has a unique expansion of the form

$$
g(t)=\underset{N \rightarrow \infty}{\lim . m} \sum_{-N}^{N} c_{n} e^{i \lambda_{n} t} .
$$

Moreover, $A \sum\left|c_{n}\right|^{2} \leq\|g\|_{L^{2}(-\pi, n)}^{2} \leq B \sum\left|c_{n}\right|^{2}$, where $A$ and $B$ are positive constants independent of $f$.

Proof. It follows from Lemma 1 that the inequality

$$
A \sum\left|c_{n}\right|^{2} \leq\left\|\sum c_{n} e^{i \lambda_{n} t}\right\|_{L^{2}(-\pi, \pi)}^{2}
$$

holds for some $A>0$ and all finite sequences $\left\{c_{n}\right\}$. Since $\left\{\lambda_{n}\right\}$ is separated (Proposition 1), Lemma 3 shows that

$$
\sum\left|f\left(\lambda_{n}\right)\right|^{2} \leq B\|f\|^{2}
$$

for some $B>0$ and every $f$ in $H$.

If $K_{n}$ denotes the reproducing function at $\lambda_{n}$,

$$
K_{n}(z)=K\left(z, \lambda_{n}\right)=\frac{\sin \pi\left(z-\bar{\lambda}_{n}\right)}{\pi\left(z-\bar{\lambda}_{n}\right)},
$$


then (15) may be rewritten as

$$
\sum\left|\left(f, K_{n}\right)\right|^{2} \leq B\|f\|^{2} .
$$

Let us set $f=\sum c_{n} K_{n}$ where $\left\{c_{n}\right\}$ is a finite sequence. By (16) and the CauchySchwarz inequality

$$
\begin{aligned}
\|f\|^{2} & =\left(f, \Sigma c_{n} K_{n}\right)=\sum \bar{c}_{n}\left(f, K_{n}\right) \\
& \leq\left[\Sigma\left|c_{n}\right|^{2}\right]^{1 / 2}\left[\Sigma\left|\left(f, K_{n}\right)\right|^{2}\right]^{1 / 2} \\
& \leq\left.\left.|\Sigma| c_{n}\right|^{2}\right|^{1 / 2} B^{1 / 2}\|f\|,
\end{aligned}
$$

so that $\|f\|^{2} \leq B \sum\left|c_{n}\right|^{2}$. Taking the Fourier transform of $f$ we get

$$
A \sum\left|c_{n}\right|^{2} \leq\left\|\sum c_{n} e^{i \lambda_{n} t}\right\|_{L^{2}(-n, \pi)}^{2} \leq B \sum\left|c_{n}\right|^{2}
$$

for every finite sequence $\left\{c_{n}\right\}$ and hence for every sequence in $l^{2}$. It is a simple consequence of (17) that each function in $L^{2}(-\pi, \pi)$ which lies in the closed linear span of $\left\{e^{i \lambda_{n} t}\right\}$ has a unique expansion of the form li.m.m. ${ }_{N \rightarrow \infty} \sum_{-N}^{N} c_{n} e^{i \lambda_{n} t}$ with $\left\{c_{n}\right\}$ in $l^{2}$. Since the exponentials $e^{i \lambda_{n} t}$ are complete in $L^{2}(-\pi, \pi)$ the result follows.

Theorem 6. Let $\left\{\lambda_{n}\right\}$ be a complete interpolating sequence. There exist positive numbers $\delta_{n}$ such that $\left\{\mu_{n}\right\}$ is a complete interpolating sequence whenever $\left|\lambda_{n}-\mu_{n}\right|$ $<\delta_{n}$.

Proof. Since $\left\{\lambda_{n}\right\}$ is interpolating there is a constant $A>0$ such that

$$
A \sum\left|c_{n}\right|^{2} \leq\left\|\sum c_{n} e^{i \lambda_{n} t}\right\|_{L^{2}(-\pi, \pi)}^{2}
$$

for every finite sequence $\left\{c_{n}\right\}$. If $\delta_{n}>0$ is chosen small enough so that

$$
\sum_{n=1}^{\infty}\left\|e^{i \lambda_{n} t}-e^{i m_{n} t}\right\|_{L^{2}(-\pi, \pi)}^{2} \leq \frac{A}{2}
$$

whenever $\left|\lambda_{n}-\mu_{n}\right|<\delta_{n}(n=1,2, \ldots)$, then for every finite sequence $\left\{c_{n}\right\}$

$$
\begin{aligned}
\left\|\sum c_{n}\left(e^{i \lambda_{n} t}-e^{i m_{n} t}\right)\right\|_{L^{2}(-n, \pi)}^{2} & \leq\left[\Sigma\left|c_{n}\right|\left\|e^{i \lambda_{n} t}-e^{i \mu_{n} t}\right\|\right]^{2} \\
& \leq\left[\Sigma\left|c_{n}\right|^{2}\right]\left[\sum\left\|e^{i \lambda_{n} t}-e^{i m_{n} t}\right\|^{2}\right] \\
& \leq \frac{A}{2} \sum\left|c_{n}\right|^{2} .
\end{aligned}
$$

Combining (18) and (19) we get

$$
\left\|\sum c_{n}\left(e^{i \lambda_{n} t}-e^{i \mu_{n} t}\right)\right\|_{L^{2}(-\rightarrow, \pi)}^{2} \leq \frac{1}{2}\left\|\sum c_{n} e^{i \lambda_{n} t}\right\|_{L^{2}(-\pi, \pi)}^{2}
$$

whenever $\left|\lambda_{n}-\mu_{n}\right|<\delta_{n}(n=1,2, \ldots)$. Since $\left\{e^{i \lambda_{n} t}\right\}$ is complete in $L^{2}(-\pi, \pi)$, it follows from a theorem of Boas [2, p. 469] that $\left\{e^{i m_{n} t}\right\}$ is also complete. In Theorem 3 it was shown that $\left\{\mu_{n}\right\}$ is interpolating whenever the $\lambda_{n}$ are sufficiently small, whence the result follows. 
4. Interpolation in $E_{\tau}^{p}$. We use the standard notation $E_{\tau}^{p}$ to denote the space of entire functions of exponential type $\tau(0<\tau<\infty)$ which belong to $L^{p}(-\infty, \infty)$ on the real axis. For the properties of the spaces $E_{\tau}^{p}$ see [4]. For $0<p<\infty$, let

$$
\|f\|_{p}=\left[\int_{-\infty}^{\infty}|f(x)|^{p} d x\right]^{1 / p},
$$

while for $p=\infty$, let $\|f\|_{\infty}=\sup |f(x)|(x$ real $)$.

Definition. A sequence $\left\{\lambda_{n}\right\}$ of distinct complex numbers is called an interpolating sequence for $E_{\tau}^{p}$ if $T E_{\tau}^{p} \supset l^{p}$. Here we continue to denote by $T$ the mapping $f \rightarrow\left\{f\left(\lambda_{n}\right)\right\}$.

The following results are derived from Lemmas 2 and 3 in essentially the same way as Proposition 1 and Corollary 1 . The proofs are therefore omitted.

Proposition 3. Let $\left\{\lambda_{n}\right\}$ be a sequence of points lying in a strip parallel to the real axis. If $T E_{\tau}^{p} \supset l^{1}(1 \leq p \leq \infty)$, then $\left\{\lambda_{n}\right\}$ is separated.

Corollary 2. Let $\left\{\lambda_{n}\right\}$ be a sequence of points lying in a strip parallel to the real axis. If $T E_{\tau}^{p} \supset l^{1}(1 \leq p \leq \infty)$, then $T E_{\tau}^{p} \subset l^{p}$.

The remainder of this section is devoted to interpolation in $E_{\tau}^{p}$ in the special cases $p=1$ and $p=\infty$.

Theorem 7. Let $\left\{\lambda_{n}\right\}$ be a sequence of points lying in a strip parallel to the real axis and suppose that there exist functions $f_{n}$ in $E_{\mu}^{\infty}$ satisfying

$$
f_{n}\left(\lambda_{k}\right)=\delta_{n k}, \quad\left\|f_{n}\right\|_{b_{0}} \leq M, \quad(\text { all } n, k) .
$$

Then $T E_{\tau}^{\infty}=l^{\infty}$ whenever $\tau>\mu$.

Proof. It is well known [4, p. 82] that for every $f$ in $E_{\tau}^{\infty}$

$$
|f(x+i y)| \leq\|f\|_{\infty} e^{\tau|y|},
$$

so that $T E_{\tau}^{\infty} \subset l^{\infty}$ for each $\tau>0$.

Fix $\left\{c_{n}\right\}$ in $l^{\infty}, \tau>\mu$ and let $\varepsilon=(\tau-\mu) / 2$. We show that the function

$$
f(z)=\sum_{-\infty}^{\infty} c_{n} f_{n}(z)\left[\frac{\sin \varepsilon\left(z-\lambda_{n}\right)}{\varepsilon\left(z-\lambda_{n}\right)}\right]^{2}
$$

belongs to $E_{\tau}^{\infty}$. Clearly, $f\left(\lambda_{n}\right)=c_{n}(-\infty<n<\infty)$. Let $\lambda_{n}=\alpha_{n}+i \beta_{n}$ and suppose that $\left|\beta_{n}\right| \leq \alpha$ and $\left|c_{n}\right| \leq N$. For $m=0,1,2, \ldots$, let $S_{m}$ be the set of integers $n$ for which $m-1 \leq\left|\lambda_{n}\right| \leq m+2$ and $T_{m}$ the set of $n$ for which $\left|\lambda_{n}\right|<m-1$ or $\left|\lambda_{n}\right|>m+2$. The method of proof of Proposition 1 shows that $\left\{\lambda_{n}\right\}$ is separated. Since $\left\{\beta_{n}\right\}$ is bounded there is a constant $K$, independent of $m$, such that the number of integers in $S_{m}$ is at most $K$. For $m \leq|z| \leq m+1$, write

$$
f(z)=\sum_{n \in S_{m}} c_{n} f_{n}(z)\left[\frac{\sin \varepsilon\left(z-\lambda_{n}\right)}{\varepsilon\left(z-\lambda_{n}\right)}\right]^{2}+\sum_{n \in T_{m}} c_{n} f_{n}(z)\left[\frac{\sin \varepsilon\left(z-\lambda_{n}\right)}{\varepsilon\left(z-\lambda_{n}\right)}\right]^{2} \text {. }
$$


Since $(\sin z) / z$ is entire of exponential type 1 and is bounded by 1 on the real axis, (20) shows that $|(\sin z) / z| \leq e^{|\operatorname{lm} z|}$. Therefore, setting $z=x+i y$, we have

$$
\begin{aligned}
|f(z)| \leq & N M \exp (\mu|y|) \sum_{n \in S_{m}} \exp \left(2 \varepsilon\left|y-\beta_{n}\right|\right) \\
& +\frac{N M \exp (\mu|y|)}{\varepsilon^{2}} \sum_{n \in T_{m}} \frac{\exp \left(2 \varepsilon\left|y-\beta_{n}\right|\right)}{\left|z-\lambda_{n}\right|^{2}} \\
\leq & K M N \exp ((\mu+2 \varepsilon)|y|+2 \varepsilon \alpha) \\
& +\varepsilon^{-2} N M \exp ((\mu+2 \varepsilon)|y|+2 \varepsilon \alpha) \sum_{n \in I_{m}} \frac{1}{\left|z-\lambda_{n}\right|^{2}} .
\end{aligned}
$$

We claim that the sums $\sum_{n \in T_{m}}\left|z-\lambda_{n}\right|^{-2}$ have a uniform upper bound for all $m \geq 0$ and $m \leq|z| \leq m+1$. Since $\left\{\lambda_{n}\right\}$ is a separated sequence, our assertion is immediate when each $\lambda_{n}$ is real, while in the general case the existence of an upper bound follows readily from the boundedness of $\operatorname{Im} \lambda_{n}$. It follows from (22) that the series in (21) converges uniformly in each disk $|z| \leq m(m=1,2, \ldots)$ and that, for some constant $A,|f(z)| \leq A \exp [(\mu+2 \varepsilon)|y|]$. Since $\tau=\mu+2 \varepsilon, f$ belongs to $E_{\tau}^{\infty}$ and the proof is complete.

Theorem 8. If $\left\{\lambda_{n}\right\}$ is a real sequence with $\lambda_{n+1}-\lambda_{n} \geq 1(-\infty<n<\infty)$, then $T E_{\tau}^{\infty}=l^{\infty}$ whenever $\tau>\pi$.

Proof. That $T E_{\tau}^{\infty} \subset l^{\infty}$ is clear. It follows readily from Theorem 1 that $\left\{\lambda_{n}\right\}$ is an interpolating sequence for $E_{\mu}^{2}$ whenever $\mu>\pi$. Indeed, if we set $\mu_{n}$ $=(\mu / \pi) \lambda_{n}$, then $\mu_{n+1}-\mu_{n} \geq \mu / \pi>1$ and Theorem 1 shows that $\left\{\mu_{n}\right\}$ is an interpolating sequence for $E_{\pi}^{2}$. Therefore, given $\left\{c_{n}\right\} \in l^{2}$ there exists a function $g$ in $E_{\pi}^{2}$ such that $g\left(\mu_{n}\right)=c_{n}$ for all $n$. Setting $f(z)=g((\mu / \pi) z)$ we see that $f$ belongs to $E_{\mu}^{2}$ and $f\left(\lambda_{n}\right)=c_{n}$ (all $n$ ), and this establishes our assertion. Let us now fix $\mu$ with $\pi<\mu<\tau$. Lemma 2 shows that there exist functions $f_{n}$ in $E_{\mu}^{2}$ for which

$$
f_{n}\left(\lambda_{k}\right)=\delta_{n k}, \quad \sup _{n}\left\|f_{n}\right\|_{2}<\infty, \quad(\text { all } n, k) .
$$

From (7) it follows that $|f(x)|^{2} \leq(\mu / \pi)\|f\|_{2}^{2}$ for all $f$ in $E_{\mu}^{2}$ and all real $x$, so that $\sup _{n}\left\|f_{n}\right\|_{\infty}<\infty$. The conclusion now follows from Theorem 7 .

In the same way we get the following result.

Theorem 9. Let $\left\{\lambda_{n}\right\}$ be a sequence of points lying in a strip parallel to the real axis and suppose that

$$
\left|\operatorname{Re}\left(\lambda_{n}\right)-n\right| \leq L<(\log 2) / \pi \quad(-\infty<n<\infty) .
$$

Then $T E_{\tau}^{\infty}=l^{\infty}$ whenever $\tau>\pi$.

Theorem 10. The integers are not an interpolating sequence for $E_{\pi}^{\infty}$.

Proof. We show that the sequence $\left\{c_{n}\right\}$ given by 


$$
\begin{aligned}
c_{n} & =0, & & n \leq 0, \\
& =(-1)^{n}, & & n>0,
\end{aligned}
$$

cannot be interpolated. Suppose first that $\left\{w_{n}\right\}$ is an arbitrary sequence in $l^{\infty}$ and that $w_{0}=0$. If there is an $f$ in $E_{\pi}^{\infty}$ with $f(n)=w_{n}(-\infty<n<\infty)$, then $g(z)=f(z) / z$ is in $E_{\pi}^{2}$ and $n g(n)=w_{n}$. If $h$ is any other function in $E_{\pi}^{2}$ for which $z h(z)$ belongs to $E_{\pi}^{\infty}$ and $n h(n)=w_{n}(-\infty<n<\infty)$, then $h(z)=g(z)$ $+\alpha(\sin \pi z) / \pi z$ for some complex number $\alpha[4$, p. 221]. Thus

$$
f(z)=z\left[\sum_{n \neq 0} \frac{w_{n}}{n} \frac{\sin \pi(z-n)}{(z-n)}+\alpha \frac{\sin \pi z}{\pi z}\right]
$$

is the most general function in $E_{\pi}^{\infty}$ with $f(n)=w_{n}(n \neq 0)$ and $f(0)=0$.

A necessary condition that $z g(z)$ belong to $E_{\pi}^{\infty}$ is that its derivative $z g^{\prime}(z)$ $+g(z)$ be bounded on the real axis $[4$, p. 206] and hence, in particular, that $n g^{\prime}(n)+g(n)$ be bounded uniformly in $n$. Since $g$ belongs to $E_{\pi}^{2}, g(n) \rightarrow 0$ as $|n| \rightarrow \infty$, so that $\left\{n g^{\prime}(n)\right\}$ must be bounded.

Now, let $\left\{c_{n}\right\}$ be given by (23) and let

$$
g(z)=\sum_{n=1}^{\infty} \frac{(-1)^{n}}{n} \frac{\sin \pi(z-n)}{\pi(z-n)} .
$$

We will show that $z g(z)$ is not bounded on the real axis by showing that $\left|n g^{\prime}(n)\right| \rightarrow \infty$ as $n \rightarrow \infty$. By the preceding remarks the integers cannot be interpolating for $E_{\pi}^{\infty}$.

We have

$$
g^{\prime}(z)=\sum_{n=1}^{\infty} \frac{1}{n} \frac{\pi^{2}(z-n) \cos \pi z-\pi \sin \pi z}{\pi^{2}(z-n)^{2}}
$$

so that for $k>0$,

$$
g^{\prime}(k) \sum_{n=1 ; n \neq k}^{\infty} \frac{1}{n} \frac{\cos \pi k}{k-n}=\sum_{n=1 ; n \neq k}^{\infty} \frac{1}{n} \frac{(-1)^{k}}{k-n} .
$$

Thus

$$
k g^{\prime}(k)=(-1)^{k} \sum_{r=1 ; n \neq k}^{\infty}\left(\frac{1}{n}-\frac{1}{n-k}\right) .
$$

It is not difficult to show that

$$
\sum_{n=1 ; n \neq k}^{\infty}\left(\frac{1}{n}-\frac{1}{n-k}\right)=\left(1+\frac{1}{2}+\cdots+\frac{1}{k}\right)-\frac{2}{k}
$$

so that $\left|k^{\prime}(k)\right| \rightarrow \infty$ as $k \rightarrow \infty$.

Theorem 11. If $\left\{\lambda_{n}\right\}$ is a real sequence with $\lambda_{n+1}-\lambda_{n} \geq 1(-\infty<n<\infty)$, then $T E_{\tau}^{1}=l^{1}$ whenever $\tau>\pi$. 
Proof. Lemma 3 shows that $T E_{\tau}^{1} \subset l^{1}$. It follows just as in the proof of Theorem 8 that for $\pi<\mu<\tau$ there exist functions $g_{n}$ in $E_{\mu}^{2}$ with $g_{n}\left(\lambda_{k}\right)=\delta_{n k}$ and $\sup _{n}\left\|g_{n}\right\|_{2}<\infty$. If we set $\varepsilon=\tau-\mu$ and let

$$
f_{n}(z)=g_{n}(z)\left(\sin \varepsilon\left(z-\lambda_{n}\right)\right) / \varepsilon\left(z-\lambda_{n}\right),
$$

then $f_{n} \in E_{\tau}^{1}$ and $f_{n}\left(\lambda_{k}\right)=\delta_{n k}$. Hölder's inequality shows that

$$
\left\|f_{n}\right\|_{1} \leq\left\|g_{n}\right\|_{2}\left\|\frac{\sin \varepsilon\left(z-\lambda_{n}\right)}{\varepsilon\left(z-\lambda_{n}\right)}\right\|_{2}
$$

and it follows that $\sup _{n}\left\|f_{n}\right\|_{1}<\infty$.

Now, choose $\left\{c_{n}\right\}$ in $l^{1}$ and set

$$
f(z)=\sum_{-\infty}^{\infty} c_{n} f_{n}(z)
$$

Since $\Sigma\left\|c_{n} f_{n}\right\|_{1}<\infty, f$ belongs to $E_{\tau}^{1}$, and Lemma 3 implies that the convergence in (24) is uniform in each horizontal strip. Therefore, $f\left(\lambda_{n}\right)=c_{n}(-\infty<n$ $<\infty)$ and the proof is complete.

It is easy to see that this result is best possible, in the sense that $\tau$ cannot always be taken equal to $\pi$. Indeed, the integers are not an interpolating sequence for $E_{\pi}^{1}$ for the trivial reason that the nonzero integers are a set of uniqueness. However, we have the following stronger result.

Theorem 12. The nonzero integers are not an interpolating sequence for $E_{\tau}^{1}$.

Proof. Lemma 3 shows that point evaluations are continuous linear functionals on $E_{\tau}^{1}$. By Lemma 2 it is enough to show that the unit ball of $l^{1}$ cannot be interpolated in a uniformly bounded way. Since

$$
f_{n}(z)=n(\sin \pi(z-n)) / \pi z(z-n) \quad(n \neq 0)
$$

is the unique function in $E_{\pi}^{1}$ with the property that $f_{n}(k)=\delta_{n k}$, it is sufficient to show that $\left\|f_{n}\right\|_{h} \rightarrow \infty$ as $n \rightarrow \infty$. For $n>0$,

$$
\begin{aligned}
\left\|f_{n}\right\|_{1} & =\int_{-\infty}^{\infty}\left|f_{n}(x)\right| d x>\frac{n}{\pi} \int_{1}^{\infty}\left|\frac{\sin \pi x}{x(x+n)}\right| d x \\
& >\frac{n}{\pi} \sum_{k=1}^{\infty} \int_{k+1 / 4}^{k+3 / 4}\left|\frac{\sin \pi x}{x(x+n)}\right| d x \\
& >\frac{\sqrt{2}}{2 \pi} \sum_{k=1}^{\infty} \int_{k+1 / 4}^{k+3 / 4}\left(\frac{1}{x}-\frac{1}{x+n}\right) d x \\
& =\frac{\sqrt{2}}{2 \pi} \log \prod_{k=1}^{\infty}\left[\frac{k+3 / 4}{k+n+3 / 4} \frac{k+n+1 / 4}{k+1 / 4}\right] .
\end{aligned}
$$

Using the relation $\Gamma(x+1)=x \Gamma(x)$ it easily follows that the infinite product 
above is equal to

$$
\begin{aligned}
\lim _{N \rightarrow \infty}\left[\frac{(1+3 / 4)(2+3 / 4) \cdots(N+3 / 4)}{(1+n+3 / 4)(2+n+3 / 4) \cdots(N+n+3 / 4)}\right] \\
\cdot\left[\frac{(1+n+1 / 4) \cdots(N+n+1 / 4)}{(1+1 / 4) \cdots(N+1 / 4)}\right] \\
=\left[\frac{1+3 / 4}{1+1 / 4}\right]\left[\frac{2+3 / 4}{2+1 / 4}\right] \cdots\left[\frac{n+3 / 4}{n+1 / 4}\right] \\
=\left[\frac{\Gamma(1+1 / 4)}{\Gamma(1+3 / 4)}\right]\left[\frac{\Gamma(n+1+3 / 4)}{\Gamma(n+1+1 / 4)}\right] .
\end{aligned}
$$

From the estimate $\Gamma(x+1) \sim(2 \pi)^{1 / 2} x^{x+1 / 2} e^{-x}($ as $x \rightarrow \infty)$ we conclude that $\Gamma(n+1+3 / 4) / \Gamma(n+1+1 / 4) \rightarrow \infty$ (as $n \rightarrow \infty)$, and the proof is complete.

The proof of the next theorem is similar to that of Theorem 11 and is therefore omitted.

Theorem 13. Let $\left\{\lambda_{n}\right\}$ be a sequence of points lying in a strip parallel to the real axis and suppose that

$$
\left|\operatorname{Re}\left(\lambda_{n}\right)-n\right| \leq L<(\log 2) / \pi \quad(-\infty<n<\infty) .
$$

Then $T E_{\tau}^{1}=l^{1}$ whenever $\tau>\pi$.

\section{BIBLIOGRAPHY}

1. N. K. Bari, Biorthogonal systems and bases in Hilbert space, Moskov. Gos. Univ. Uren. Zap. 148 Mat. 4 (1951), 69-107. (Russian) MR 14, 289.

2. R. P. Boas, Jr., Expansions of analytic functions, Trans. Amer. Math. Soc. 48 (1940), 467-487. MR $2,80$.

3. - A general moment problem, Amer. J. Math. 63 (1941), 361-370. MR 2, 281.

4. - Entire functions, Academic Press, New York, 1954. MR 16, 914.

5. R. J. Duffin and J. J. Eachus, Some notes on an expansion theorem of Paley and Wiener, Bull. Amer. Math. Soc. 48 (1942), 850-855. MR 4, 97.

6. R. J. Duffin and A. C. Schaeffer, A class of nonharmonic Fourier series, Trans. Amer. Math. Soc. 72 (1952), 341-366. MR 13, 839.

7. A. E. Ingham, Some trigonometrical inequalities with applications to the theory of series, Math. $\mathbf{Z}$. 41 (1936), 367-379.

8. R. Paley and N. Wiener, Fourier transforms in the complex domain, Amer. Math. Soc. Colloq. Publ., vol. 9, Amer. Math. Soc., Providence, R.I., 1934.

9. J. Rosenbaum, Interpolation in Hilbert spaces of analytic functions, Thesis, University of Michigan, Ann Arbor, Mich., 1965.

10. H. S. Shapiro and A. L. Shields, On some interpolation problems for analytic functions, Amer. J. Math. 83 (1961), 513-532. MR 24 \# A3280.

11. E. C. Titchmarsh, The zeros of certain integral functions, Proc. London Math. Soc. (2) 25 (1926), 283-302.

Department of Mathematics, Oberlin College,Oberlin, Oho 44074 